\title{
$\mathrm{XX}$
}

\section{Zur Kenntniss der centralen Hämatomyelie.}

\author{
Von \\ Dr. E. Bregman, \\ ord. Arzt der Nervenklinik in Warschan.
}

(Mit 2 Abbildungen im Text.)

Als centrale Hämatomyelie bat bekanntlich Minor ${ }^{1}$ ) diejenigen Fälle zu bezeichnen vorgeschlagen, in welchen sich neben Muskelatrophien und Paresen eine partielle Empfindungslähmung, ähnlich wie bei Syringomyelie, vorfindet und auf Grund all dieser Symptome eine Localisation der Blutung innerbalb der grauen Substanz voransgesetzt werden darf. Nach der Vertheilung der einzelnen Symptome lassen sich diese Fälle in 3 Typen theilen, welche aber auch combinirt vorkommen können.

1. Fälle mit Brown-Séquard'sehem Typus - also Lähmung auf einer Seite, Sensibilitätsstörung auf der anderen.

2. In den Armen Paresen mit Atrophie, in den Beinen Paresen ohne Atropbie; Empfindungslähmung am Rumpf und in den Beinen.

3. Motorische und sensible Lähmung auf derselben Seite, wie bei der Syringomyelie.

Ich möchte mir nun erlauben, die Krankengeschichte eines Falles mitzutheilen, welche zur centralen Hämatomyelie gezählt werden darf, und zwar eine Combination der Typen 1 und 3 vorstellt. Die Erörterung dieses Falles wird mir dann Veranlassung geben, auf einige Punkte in der Diagnose und Pathologie dieses Leidens etwas näher einzugehen.

C b. M. wurde am 29. Juni 1895 in die therapentisehe Klinik aufgenommen. Pat., 30 Jahre alt, verheirathet, war vor seiner gegenwärtigen Erkrankung immer gesund, hatte weder Lues, noch irgend eine andere venerische Erkrankung durchgemacht, kein Abusus spirituosum. In einem Ledergeschäft beschäftigt, hatte Pat. schwere Lasten auf dem Rücken zu tragen. Auch am Tage der Erkrankung musste er ca. 10 Felle

1) Archiv für Psychiatrie. Bd. XXIV. Heft 3 und Bd. XXVIII. Heft 1. 
auf dem Rücken schleppen (immerhin noch nicht das Maximum seiner Leistungen), fühlte aber unmittelbar daranf keine irgend welche $\mathrm{Be}$ schwerden. Irgend eine andere Ursache für seine Erkrankung weiss er nicht anzugeben, erwähnenswerth wäre vielleicht nur, dass er Nachts vor derselben sich geschlechtlich stark aufgeregt hatte, obne dass er, wegen äusserer Hindernisse, im Stande war, den Coitus auszuüben.

Seine Erkrankung begann damit, dass er plötzlich, gegen Mittag etwa 21/2. Monate vor Eintritt in die Klinik - heftige Schmerzen im Riicken empfand, welche ihn veranlassten, sich sofort in's Bett zu legen. Es gesellten sich bald Schmerzen im Abdomen binzu, und zugleich fühlte Pat. das Bedurfniss zum Harnlassen, konnte aber seinen Urin nicht entleeren. Das linke Bein war vollkommen gelähmt; keine Schmerzen daselbst. Der herbeigerufene Arzt hat die Harnblase durch den Catheter entleert. Die Harnretention dauerte noch ca. 2 Tage, Pat. wurde einige Male catheterisirt. Darauf stellte sich Incontinenz ein, und sehr bald ersehien der Harn blutig gefärbt uud mit viel Eiter gemischt, ja manchmal soll reiner Eiter aus der Harnröhre geflossen sein. Späterhin vermochte Pat. den Harn zurückzuhalten, das Harnlassen war aber erschwert, es dauerte lange bis der Harn berauskam: diese Beschwerden hatten sich bis zur Aufnahme in die Klinik nur wenig vermindert. Beim Harnlassen starke Schmerzen am Orificium urethrae und in der Gegend der Symphysis. Die Menge des Eiters im Urin ist allmählich geringer geworden. Die Kraft der linken unteren Extremität besserte sich allmählich, nach 3 Wochen bereits machte Pat. die ersten Gehversuche. Pat. wurde in Lublin mit KJ, Strychnin und Bädern behandelt.

Status praesens 29. Juni 1895. Pat. ist mittleren Wuchses, von gracilem Körperbau, Haut und Schleimhäute blass. Innere Organe normal, Körpertemperatur meist erhöht, Puls 80 , regelmässig.

Von Seiten der Hirnnerven keine Störung. Die Wirbelsäule zeigt keine Gestaltveränderung, der Dornfortsatz des ersten Lendenwirbels etwas druckempfindlich.

In den oberen Extremitäten keine Störung. Beim Gehen wird das linke Bein nachgeschleppt. Stehen auf dem rechten Bein gut, auf dem linken unmöglich. Grobe Kraft links bedentend und gleichmässig herabgesetzt. Keine sichtbare Atrophie der linken unteren Extremitäten. Circumferenz der Wade r. $34,1.33$; - des Oberschenkels $(15 \mathrm{Cm}$. über der Patella) $\mathrm{r} .=1 .=45$. Muskeltonus gegen rechts nicht dentlich verschieden.

Kniereflex links bedeutend erhöht, rechts annähernd normal, desgleichen die Achillessehnenreflexe. Links starker Fussclonus, rechts keiner. Fusssohlenreflex links mässig, rechts abwesend, gleichfalls Cremasterreflexe.

Tactile Empfindung und Ortssinn überall gut erhalten, dagegen Schmerzund Temperatursinn in gewissen Theilen vollkommen erloschen, und zwar erstreckt sich diese Sensibilitätsstörung (vgl. Fig. 1 und 2): rech terseits auf die ganze untere Extremität und die untere Rumpfhälfte etwa bis zur Höhe des Proe. syphoideus (wobei jedoch oberhalb des Nabels die Empfindung. nicht vollkommen aufgehoben sich erweist), linkerseits auf die untere Rumpfhälfte und einen Theil der vorderen Schenkelfläche; 
auch hier ist an der oberen und unteren Grenze dieses Gebietes der Empfindungsverlust unvollständig (Pat. giebt nun mit Bestimmtheit an, dass er vom Beginn an am rechten Fuss die Wärme- und Kälteempfindung verloren, am linken behalten hatte). Eine hyperästhetische Zone nicht nachweisbar. Muskelsinn beiderseits normal. Die faradocutane Schmerz-

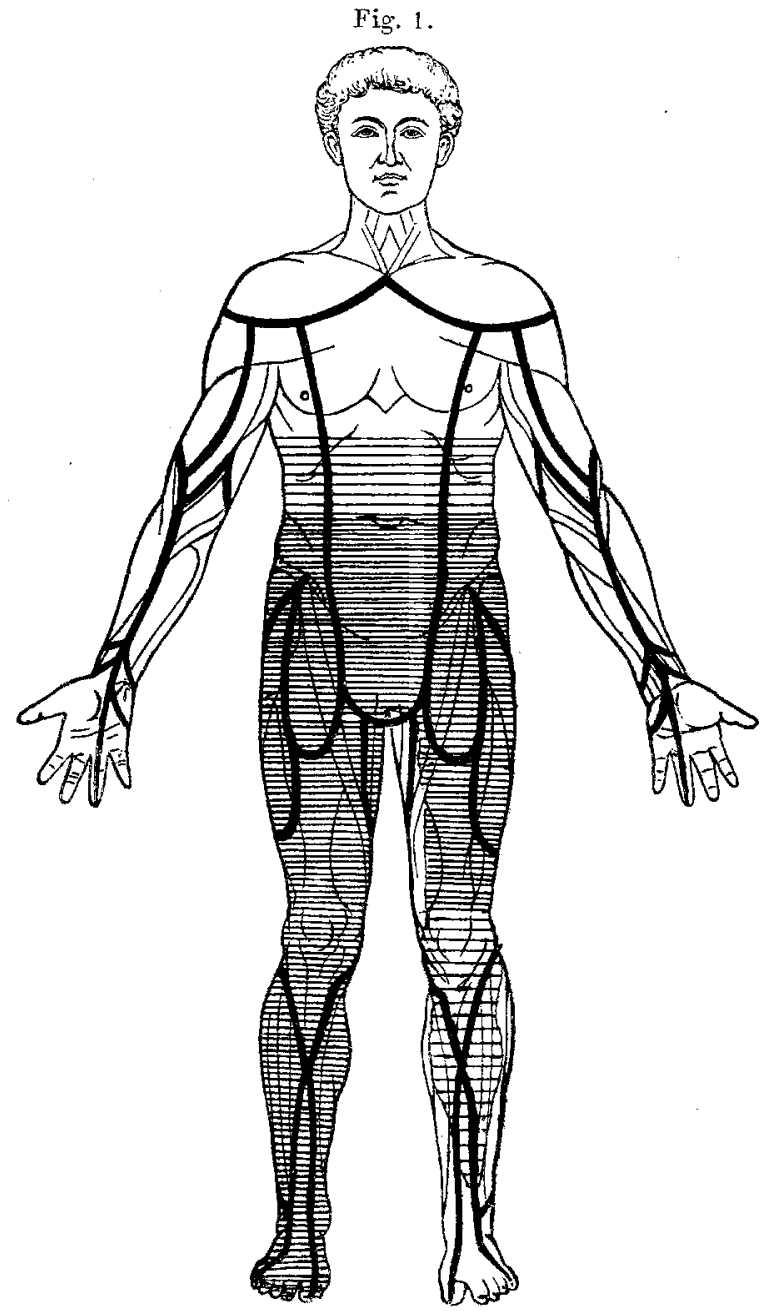

empfindung zeigt sich am linken Unterschenkel bei $5 \mathrm{Cm}$. RA, rechts anch nicht bei 0 . Die faradische Muskelerregbarkeit unverändert.

Der Harn trübe, von saurer Reaction, enthält weder Eiweiss, noch Zucker, im Absatz fast ausschliesslich Eiterkörperchen, sehr spärliche rothe Blutkörperchen.

Pat. verblieb in der Klinik bis zum 8. Juli. In seinem Zustande 
trat keiue wesentliche Aenderung ein, nur am 7. Juli zeigten sich links in der Höhe des Proc. xyphoideus einige Herpesbläschen : keine Schmerzen an dieser Stelle, nur leichtes Jucken.

Anfangs März d. J. hatte ich wieder Gelegenheit, den Kranken zu untersuchen (am 2. März habe ich ihn in der Warschauer Gesell.

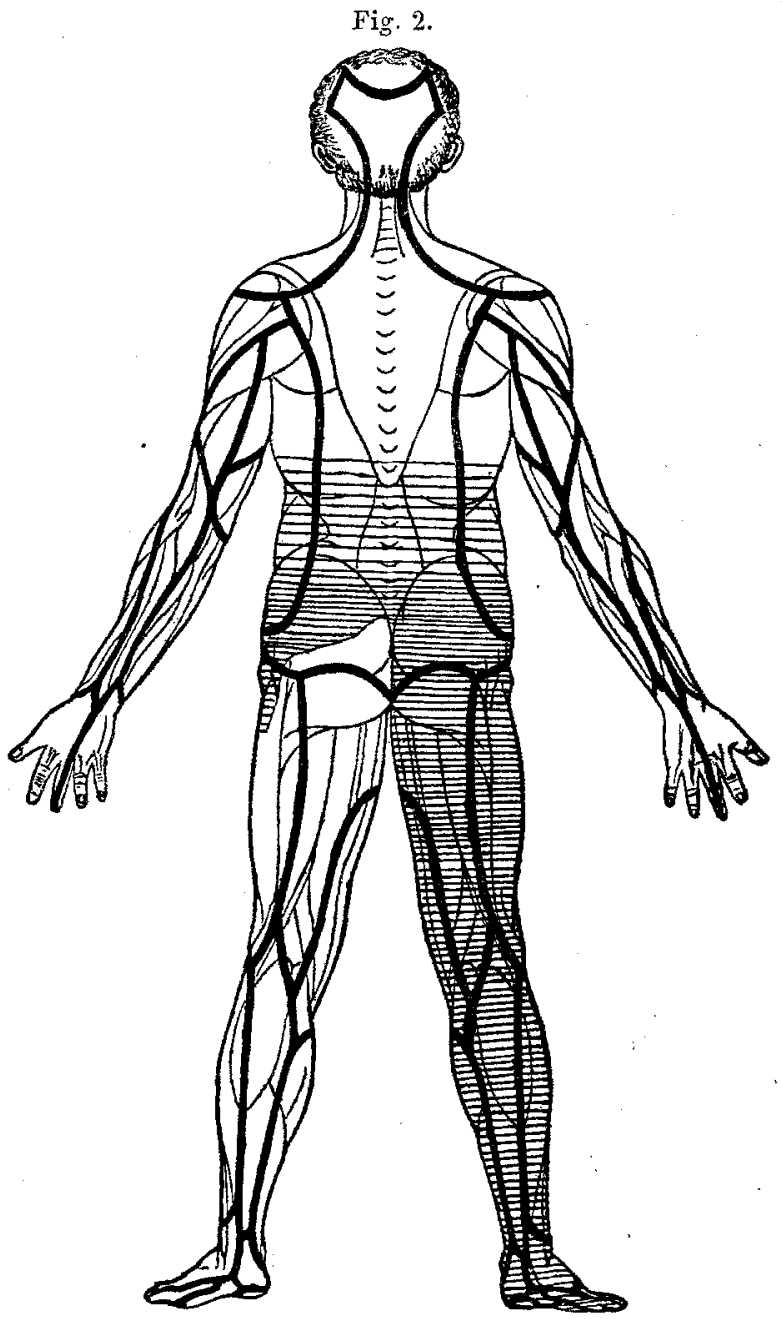

schaft der Aerzte demonstrirt): die Parese der linken unteren Extremität hat sich bedeutend gebessert, so dass beim Gehen nur ein leichtes Hinken bemerbar wird. Stehen auf dem linken Bein fällt dem Kranken schwer. Die Sehnenreflexe links bedeutend gesteigert, starker Fussclonus. Die Sensibilitätsstörungen bestehen unverändert fort. Parästhesien in der linken Lende und an der Vorderfläche des linken Oberschenkels Deutsche Zeitschr. f. Nervenheilkunde. X. Bd. 
(Taublheitsgefühl) dauern fort. Auch die Druckempfindlichkeit des Dornfortsatzes des ersten Lendenwirbels ist geblieben. Eine gewisse Schwierigkeit beim Harnlassen besteht noch. Neigung zur Verstopfung. Potenz erhalten, jedoch geschlechtliche Befriedigung mangelhaft.

Kurz zusammenfassend hatten wir bei einem 30 jährigen Lederhändler neben einer spastischen Parese der linken unteren Extremität eine partielle (syringomyelische) Empfindungslähmung der rechten unteren Extremität und der rechten unteren Rumpfhälfte, ferner eine gleiche Empfindungsstörung am Rumpfe links und auf der Vorderfläcbe des linken Schenkels. Die Erkrankung begann plötzlich, mit starken initialen Schmerzen im Rücken und Abdomen, obne irgend welche unmittelbare Veranlassung, nachdem jedoch Pat. kurz zuvor, wie gewöhnlich, schwere Lasten auf dem Rücken getragen hatte. Zugleich mit der Läbmung stellten sich Blasenstörungen ein. Sebr früb kam es zur Entwicklung einer Cystitis. Im Laufe der folgenden 2 Jahre Regression aller Erscheinungen mit Ausnahme der Sensibilitätsstörungen. Auf Grund dieser Daten erscheint die Diagnose einer intramedullären Blutung als die bei Weitem wahrscheinlichste. Zu Gunsten derselben sprechen vor Allem der plötzliche Beginn und der spätere Rückgang: der Symptome. Embolie und Thrombose finden sich erfahrungsgemäss in diesem Gebiete viel seltener, andererseits lassen sich dafür keine ätiologischen Momente vorfïhren, der Kranke ist jung, sein Herz normal, fïr Lues liegen keine Anhaltspunkte vor, keinerlei Erkrankung ist dem gegenwärtigen Leiden vorangegangen.

Angesichts der initialen Schmerzen und des späteren Herpes könnte an einen extramedullären Bluterguss (Hämatorrhachis) gedacht werden, indess auf Grund der Symptome einer Halbseitenläsion, des weiteren schmerzlosen Verlanfes, der geringen Druckempfindlichkeit der Wirbelsäule und Schmerzmangel bei Körperbewegungen, darf eine solche ausgeschlossen werden. Auch die Annahme einer primären Gliose, welche etwa acut in Scene trat, erscheint nach der nunmehr 2 jährigen Beobachtung in hohem Grade unwabrscheinlich.

Grössere Schwierigkeiten bietet die Differentialdiagnose gegenüber der acuten Myelitis. Der fieberlose und apoplectiforme Beginn sprechen zu Gunsten einer Hämatomyelie: zwar wurde auch bei der Myelitis ein apoplectiformer Beginn bescbrieben, jedoch kommt derartiges sehr selten vor, in der Regel braucht dieselbe mindestens 2-3 Tage, bis die Symptome ibre Höhe erreichen. Andererseits kann freilich auch die Hämatomyelie, zwar acut einsetzend, sich in der Folgezeit auf weitere Strecken ausdehnen, und somit können sich ibre Symptome noch später einer Steigerung fähig erweisen (vgl. die 


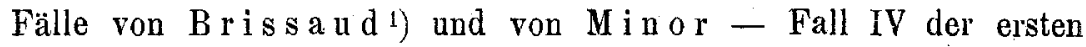
Serie).

Im Allgemeinen wird die acute Myelitis mehr Neigung zeigen, sich auf den ganzen Querschnitt auszudehnen; in solchem Falle also, wo, wie im unserigen, die Symptome denen einer Halbseitenläsion sich nähern, und die Empfindungslähmung partiell war, ist daher auch aus diesem Grunde eine Blutung viel wahrscheinlicher. Schliesslich sind die ätiologischen Verhältnisse für die Diagnose von maassgebender Bedeutung. Die Infectionskrankheiten, die bei der Myelitis acuta vor Allem in Betracht kommen ${ }^{2}$, haben wohl zar Hämatomyelie kaum irgend welche Beziehungen. Zwar wurden spärliche Fälle im Anschluss daran, namentlich an Influenza, beschrieben, da es sich jedoch nur um klinische Beobachtungen ohne Nekropsie handelte, kann die Sache dadurch nicht entschieden werden. Im Falle von Williamson ${ }^{3}$ ), wo die Diagnose Hämatomyelie durch die Section widerlegt wurde, liess sich vielleicht der Irrthum vermeiden, wenn die Aetiologie mehr Berücksichtigung gefunden hätte (Typhus ging dem Leiden voran).

Dagegen kommt einem anderen Moment bei der Hämatomyelie eine grosse Bedeutung zu, nämlich dem Trauma. Wir finden dasselbe in den meisten Fällen, in welchen überhaupt eine Ursache nachgewiesen werden konnte. In unserem Falle lag gleichfalls eine traumatische Einwirkung vor, welche aber von dem Beginn der Erkrankung durch ein paar Stunden getrennt war und zu der gewohnten Beschäftigung des Kranken gehörte. Wir glauben, dass man deshalb diesem Umstande seine ätiologische Bedeutung nicht absprechen darf, um so mehr, als gerade dureh öftere Wiederholung derartiger Ein. wirkungen der Kreislauf innerbalb des Rückgratkanals schädlich beeiuflusst werden musste.

Es darf somit unser Fall auf Grund des Beginnes, der Symptome, des Verlaufes und der Aetiologie wohl mit Recht zur Hämatomyelie gezäblt werden, nnd steht er in nahen Beziehungen zu den von Min or als centrale Hämatomyelie ausgeschiedenen Fällen. Was nun diesen letzten Begriff betrifft, so ist es eine seit jeher bekannte Thatsache, dass die graue Substanz eine Prädilectionsstelle für Blutungen ist, und dass diese sich darin longitudinal auszudehnen pflegen. Neuer-

1) Leçons sur les maladies du système normal. 1895 .

2) Wir verweisen besonders auf die sehr bemerkenswerthen Auseinandersetzungen, welche Bruns auf der Versammlung der Irrenärzte zu Hannover (1896) vorgetragen hat.

3) The Lancet 1894, 7. July. 
dings haben Goldscheider und Flata ${ }^{1}$ ) diese Frage experimentell geprüft, indem sie menschlichen Leichen Farblösungen ins Rückenmark einspritzten. Während nun die Infiltration bei Einspritzung in die weisse Substanz sebr gering ausfiel, wurde sie sofort bedeutender, sobald in der Nähe der grauen injicirt wurde, indem die Flüssigkeit sowohl in transversaler als in longitudinaler Richtung sich in letzterer ausbreitete. Um so mehr war dies der Fall, wenn diese direct getroffen wurde, hierbei stellte sich jedoch ein bemerkenswerther Unterschied zwischen Vorder- und Hinterborn heraus: bei Injection in das erstere nämlich hatte die Flüssigkeit grosse Neigung, sich auf die uibrige homo- und contralaterale graue Substanz auszudehnen, dagegen beschränkte sie sich bei Injection in das Hinterhorn darauf, letzteres longitudinal zu durchsetzen. Ferner fiel das Ergebniss verschieden aus, je nachdem in das laterale oder in das mediale Stück des Vorderhornes injicirt wurde: im ersteren Falle erstreckte sich die Infiltration auf die anliegende weisse Substanz, im zweiten blieb diese verschont, die Flüssigkeit ergoss sich aber auf die übrigen Theile der grauen.

Inwieweit diese interessanten Ergebnisse mit den Thatsachen der menschlichen Pathologie ïbereinstimmen, dürfte schwer zu erweisen sein, da anatomisch untersuchte Fälle von Hämatomyelie in nur geringer Zahl vorliegen und andererseits der Ausgangspunkt einer ausgedehnten Blutung nicht immer mit Sicherheit festzustellen ist. Im Wesentlichen wird aber dadurch noch einmal bestätigt, dass die graue Substanz und speciell das Hinterhorn der Ausbreitung der Blutung den geringsten Widerstand entgegensetzt, andererseits wird aber auch auf die Möglichkeit einer Betheiligung der weissen Substanz hingewiesen.

Ein wichtiges Kennzeichen der centralen Hämatomyelie bildet das Symptom der partiellen Empfindungslähmung, welches man auf eine Erkrankung der hinteren Theile der grauen Substanz zu beziehen pflegt. Diese Beziehung könnte aber nưr in dem Falle als eine constante hingenommen werden, wenn erwiesen sein würde, dass die Bahnen für den Schmerz- und Temperatursinn innerhalb der grauen Substanz in die Höhe steigen, denn nur dann wäre es möglich, dass eine Läsion eines bestimmten Rückenmarkssegmentes eine derartige Störung in der ganzen darunterliegenden Körperpartie erzengt. Nun macht sich aber immer mehr die entgegengesetzte Ansicht geltend, dass die genannten Bahnen nicht in der grauen Substanz verbleiben, sondern eine andere Richtung einschlagen.

Zunächst muss betont werden, dass für jene zuerst erwähnte Voraussetzung, welche von Physiologen auf Grund ihrer experimen-

1) Zeitschrift für klin. Medicin. Bd. XXXI. H. 3 u. 4. 
tellen Ergebnisse ausgesprochen wurde, eine anatomische Begründung nicht dargebracht werden konnte: secundüre aufsteigende Degeneration, wie solche nach Unterbrechung centripetalleitender Bahnen einzntreten pflegt, konnte innerhalb der grauen Substanz nicht nachgewiesen werden. Diesem Einwand wurde damit begegnet, dass es sich um myelinlose Fasern handelt, die Degeneration aus diesem Grunde schwer erkennbar sei, und dass die betreffenden Bahnen sich vielleicht aus einer Reihe kurzer, durch Zellen unterbrochener Strecken zusammensetzen. Indess hat neuerdings $\mathrm{Ci}$ agliński ${ }^{1}$ ) auch eine aufsteigende Degeneration innerhalb der grauen Substanz behauptet: nach Unterbindung des Rückenmarkes im Lumbalabschnitte bei Hunden mittelst einer Seidenligatur fand er (nach Marcbi) einen degenerirten Strang von drei-, resp. in höheren Abschnitten viereckiger Gestalt, welcher den hinteren Theil der centralen grauen Substanz zwisehen Centralkanal und Hintersträngen einnahm und bis über die Cervicalanschwellung verfolgt werden konnte.

Da jedoch dieser Befund inmitten der grossen Anzahl dieses Gebiet betreffender Untersuchungen ganz isolirt dasteht, wird man wohl noch seine Bestätigung durch weitere Forschungen abwarten müssen, um so mehr als andere Erwägungen und Thatsachen auf einen ganz anderen Verlauf der gemachten Bahnen hinzudeuten scheinen. Zunächst die Betrachtung der Sensibilitätsstörungen bei Syringomyelie.

Bei dieser Krankheit ist die centrale graue Substanz in hervorragender Weise betheiligt, und die partielle Empfindungslähmung wird als dessen Folge betrachtet. Dieselbe erstreckt sich aber durchaus nicht auf die ganze unterhalb der Läsion gelegene Partie, sondern nur auf diejenigen Körpertheile, welche dem betroffenen Rïckenmarkssegment entsprechen. Dementsprechend findet sich in vielen Fällen die "Giletform", bedingt durch die häufigste Localisation der Gliose im unteren Hals- und oberen Brustmark. Laehr ${ }^{3}$ ) hat neuerdings die Vertheilung der Senbilitätsstörungen bei der Syringomyelie geprüft und gelangt zum Schluss, dass auch im Einzelnen die Localisation der partiellen Empfindungslähmung dem Gebiete einzelner Wurzeln, also einzelner Riickenmarkssegmente analog ist. Diese Behauptung

1) Gazeta lekarska. 1896. Nr. 10 u. 11 und Neurolog. Centralbl. 1896. Nr.17.

2) Bei anderen Hunden konnte -2 Wochen nach der Operation - keine Degeneration nachgewiesen werden. Bei einem Hunde fand sich unterhalb der Compressionsstelle eine Höhle an Stelle des Stranges. Derartige Höhlen, infolge von Nekrose, wurden bei Rückenmarkscompression vielmals beobachtet, und die Möglichkeit ist nicht von der Hand zu weisen, dass auch die angebliche Degeneration auf die gleiche Weise entstanden sei.

3) Archiv für Psychiatrie. Bd. XXVIII. H. 3. 
steht sogar in directem Gegensatze zu der seit $\mathrm{Charcot}$ geläufigen Meinung, dass nämlich die Empfindungsstörungen bei der Syringomyelie an den Extremitäten sich, ganz wie bei der Hysterie, durch Amputationslinien abgrenzen. Diese Coincidenz eines so charakteristischen Merkmales bei zwei so verschiedenen Leiden erscheint zwar von vornherein sehr wundersam, immerhin wird man angesichts der Autorität des grossen französischen Forschers mit dem endgulltigen Urtheil in dieser Sache abwarten müssen, bis weitere genaue Untersuchungen an einer grösseren Anzahl von Fällen gemacht worden sind. Wie dem aber auch sein mag, eins steht jedoch fest, das nämlich bei Syringomyelie trotz Betheiligung eines grossen Theiles der grauen Substanz, die Empfindung in den unterhalb gelegenen Theilen erhalten bleibt. Sensibilitätsstörungen in den unteren Extremitäten sind nur da vorhanden, wo auch die Gliose in longitudinaler Richtung eine grössere Ausdehnung gewonnen hat. 1) Diese Thatsache lässt sich schwer mit der Voraussetzung eines bis zum Gebirn aufsteigenden Verlaufes der sensiblen Bahnen innerhalb der grauen Substanz in Einklang bringen. Denn bei einem Zusammengedrängtsein derselben etwa auf den kleinen Raum, welcher ihnen von Ciągliński angewiesen wird, eine Partie, die thatsächlich bei diesem Leiden am häufigsten erkrankt sich findet, wäre es kaum zu begreifen, wie die von unten kommenden Fasern verschont bleiben können.

Ist somit das Aufsteigen der Schmerz- und Temperatursinnbahnen innerbalb der grauen Substanz unwahrscheinlieb, wo sollen wir dann den weiteren Verlauf derselben suchen?

Schlesinger ${ }^{2}$, welcher diese Frage im Wiener physiologischen Club discutirte, äussert sich zu Gunsten des Gowers'schen Bündels und des Vorderstranges. In einem Falle von Compressionsmyelitis fand er deutliche aufsteigende Degeneration im Vorderstrange. Laebr (l. c.) versetzt die genannten Bahnen in die Seitenstränge und hebt besonders hervor, dass die Thatsachen der menschlischen Pathologie mit dieser Annahme in vollem Einklang stehen. Für diejenigen Fälle von Hämatomyelie von Brown-Séquard'schem Typus ist die Mitbetheiligung der weissen Substanz um so wahrscheinlicher, als hier beim engen Zusammenliegen der betreffenden Fasern eine beschränkte Läsion ausgedehnte Empfindungsstörungen hervorbringen kann. Die

1) Andererseits kann auch die weisse Substanz in höherem Grade betheiligt sein, so dass davon nur ein schmaler Ring um die Rückenmarksperipberie zurückbleibt: in solchem Falle können in den tiefergelegenen Körpertheilen Sensibilitätsstörungen auftreten, trotz Intactbleiben der entsprechenden Rückenmarkssegmente.

2) Neurolog. Centralblatt. 1895. Nr. 16. 
Möglichkeit eines derartigen Uebergreifens auf die weisse Substanz haben Goldscheider und Flata auch experimentell bewiesen (s.o).

Die von Holzinger ${ }^{1}$ ) in Bechterew's Laboratorium ausgefuhrten Experimente weisen auf den hinteren Theil der Seitenstränge hin; die Zerstörung der ganzen grauen Substanz zusammen mit den Hintersträngen hatte keine Analgesie zur Folge, wenn jene Partie erbalten blieb.

Nach Man n ${ }^{2}$ ) verlaufen die genannten Bahnen nach ibrer Kreuzung in der Grenzschicht der weissen Substanz und rücken später, indem sie von den höher sich kreuzenden Fasern verdrängt werden, näher an die Peripherie in die Seitenstrangreste. Mann stützt sich auf die anatomische Untersuchung eines Falles von traumatischer Zerstörung des Rïckenmarkes, vorztiglich einer Hälfte. Im weiteren Verlauf sammeln sich wahrscheinlich alle hierhergehörige Fasern im Gowers'schen Bündel.

Eding e ${ }^{3}$ ) glaubt, dass die sensiblen Fasern nach der Kreuzung über das ganze Gebiet der Vorder- und Hinterstränge zerstreut sind, dass aber speciell das Areal der Vorderseitenstrangreste viele dieser Fasern enthält. Dieser Autor nimmt eine Unterbrechung der sensorischen Bahn in der gleichnamigen grauen Substanz an (Aufsplitterung der hinteren Wurzelfasern um Vorder- und Hinterhornzellen) mit nachfolgender Kreuzung in der vorderen Commissur; dagegen glaubt S c blesinger eine Unterbrechung nach der Kreuzung efwa im Vorderhorn voraussetzen zu dürfen, weil vollständige Zerstörung des Hinterhornes keine aufsteigende Degeneration zur Folge hatte. ${ }^{4}$ )

Wenn wir nun alles Gesagte zusammenfassen und auf unseren Fall anzuwenden versuchen, so können wir zunächst sagen; dass eine beschränkte Blutung innerhalb der grauen Substanz wohl unter keinen Umständen eine partielle Empfindungslähmung in der contralateralen Körperhälfte hervorbringen kann, da ja, wie wir sehen, die Annahme des Verbleibens der Schmerz- und Temperatursinnbahnen in der grauen Substanz nicht aufrecht gehalten werden kann. Eher schon würde eine Röhrenblutung längs einer Hälfte der grauen Substanz bis zu den untersten Rückenmarksabschnitten im Stande sein, eine derartige Störung zu erzengen, wenn wir voraussetzen, dass die betreffenden Fasern nach der Kreazung die graue Substanz passiren, was auch nach den neueren Untersuchungen nicht ausgeschlossen

1) Bechterew, Neurolog. Centralblatt. 1894. Nr. 18.

2) Deutsche Zeitschrift für Nervenheilkunde. Bd. X. H. 1 u. 2.

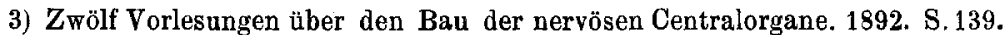

4) Vgl. dagegen die Arbeit von L. R. Müller, Deutsche Zeitschrift f. Nervenheilkunde. Bd. X. S. 289 flg. 
erscheint. Vor allem müsste freilich eine derartige Blutung, aus leicht ersichtlichen Gründen, eine Empfindungsstörung auf der Seite der Läsion zur Folge haben. In den Fällen von Hämatomyelie, welche dem syringomyelischen Typus entsprechen (Lähmung und Sensibilitätsstörung auf der gleichen Seite), sind wir sogar gezwungen, eine solche Röhrenblutung in der durch die Ausbreitung der Empfindungslähmung angezeigten Länge anzunehmen, denn keine andere Localisation vermag uns eine Erklärung der Störungen zu geben. Dagegen können die Fälle von B rown-Séquard'schem Typus nach den obigen Auseinandersetzungen viel leichter auf eine Blutung bezogen werden, welche in ihrer Höhenausdehnung zwar weniger ausgiebig, von der grauen auch auf die angrenzende weisse Substanz übergreift: allein der der Höhe der Läsion entsprechende und mit ihr gleichnamige Anästhesiestreifen müsste dann auf die Betheiligung der grauen Substanz zurückgeführt werden.

In unserem Falle hatten wir neben dem Brown-Séquard'schen Lähmungstypus eine Zone partieller Empfindungslähmung auf der Seite der Lähmung, welche um Vieles den in typischen Fällen von Halbseitenläsion vorgefundenen gleichnamigen Anästhesiestreifen übertraf. Wenn wir die Ausdehnung dieser Zone mit dem bekannten Thor burn'schen Schema vergleichen, so kann auf eine Betheiligung des Rückenmarkes vom 8-9 Brustsegmente bis etwa zum 3.-4. Lendensegmente geschlossen werden. Nach unserer Auffassung muss auf dieser Strecke eine Läsion der hinteren Hälfte der grauen Substanz angenommen werden; an irgend einer Stelle, vielleicht am Ausgangspunkte der Blutung, muss aber auch ein Theil des Seitenstranges mitzerstört worden sein, worauf die dauernde contralaterale Analgesie und Thermanästhesie bezogen werden muss. Diese Erklärung wird dadurch noch wahrscheinlicher, dass wir in der spastischen Parese der linken unteren Extremität ein weiteres Symptom besitzen, welches uns auf eine Betheiligung des linken Seitenstranges hinweist. Weiteres pathologisch anatomisches Material wäre sehr erwiunscht, nm die Localisation der Blutung in ähnlichen Fällen sicherzustellen; indess, angesichts des meist benignen Verlaufes derselben, kann solches wohl erst nach längerer Zeit angesammelt werden. Diejenigen Fälle von Hämatomyelie aber, welche rasch tödtlich verlaufen, sind meist mit anderen Läsionen - Wirbelsäulefracturen und Luxationen, Rückenmarkserschütterung und Quetschung - complicirt und werden daher in Bezug auf die Localisation der klinischen Erscheinungen nur beschränkte Anwendung finden können.

Warschau, den 17. April 1897. 